been put to better use if left for females ${ }^{5}$.

Why doesn't evolution favour asexual reproduction, to avoid the types of struggle between males and females that can have a negative effect on overall population fitness? Theoretically, an animal lineage that reproduces asexually should eventually run into difficulties. However, it was reported ${ }^{6}$ this year that Amazon molly fish (Poecilia formosa) have a genome of surprisingly good quality, even after about 500,000 generations of asexual reproduction. Many such evolutionary mysteries will provide fascinating research topics for years to come.

Martins and colleagues' approach overcomes caveats in previous attempts to measure how differences between the sexes affect population fitness. In a technique termed experimental evolution, the strength of sexual selection can be varied experimentally by restricting mating in some captive lineages to between monogamous pairs, while allowing competition for mates to operate in other lineages of the same species ${ }^{7}$. Another approach involves using information on species existing today and indicators of sexual competition, such as testis size or differences in size or colour between males and females. These indicators are then compared with the estimated risk of species extinction as documented ${ }^{8}$ in the Red List generated by the International Union for Conservation of Nature, or with the levels of population turnover ${ }^{9}$. However, experimental evolution is usually carried out in a simplified laboratory environment, whereas current threats to species persistence often have human-mediated causes. Martins and colleagues managed to show the risks of pronounced male-female differences over a long period before humans had evolved.

We can thank sexual selection for wondrous traits such as the peacock's tail, the courtship dance (Fig. 1) of the colourful male peacock spiders of the genus Maratus and, indeed, the elongated shape of male ostracods. However, as Martins et al. have shown, differences between the sexes can have negative consequences for species. With more than 10,000 ostracod species still in existence (including asexual ones), it is surprising how little we know about their genetics or other demographic factors that affect how these populations thrive, including the conditions under which they reproduce or survive well ${ }^{10}$. Why do differences between male and female ostracods result in an increase in the risk of extinction? Experimental evolution, anyone?

Hanna Kokko is in the Department of Evolutionary Biology and Environmental Studies, University of Zurich, 8057 Zurich, Switzerland.

e-mail:hanna.kokko@ieu.uzh.ch

1. Martins, M. J. F., Puckett, T. M., Lockwood, R Swaddle, J. P. \& Hunt, G. Nature 556, 366-369 (2018).

2. Whitlock, M. C. Evolution 54, 1855-1861 (2000).

3. Mank, J. E. Nature Rev. Genet. 18, 721-730 (2017)

4. Le Galliard, J.-F., Fitze, P. S., Ferrière, R. \& Clobert, J. Proc. Natl Acad. Sci. USA 102, 18231-18236 (2005).

5. Kokko, H. \& Brooks, R. Ann. Zool. Fennici 40, 207-219 (2003).

6. Warren, W. C. et al. Nature Ecol. Evol. 2, 669-679 (2018).

7. Lumley, A. J. et al. Nature 522, 470-473 (2015).

8. Morrow, E. H. \& Fricke, C. Proc. R. Soc. Lond. B 271, 2395-2401 (2004).

9. Doherty, P. F. et al. Proc. Natl Acad. Sci. USA 100, 5858-5862 (2003).

10.Park, A. W., Vandekerhove, J. \& Michalakis, Y. J. Evol. Biol. 27, 1650-1661 (2014).

This article was published online on 11 April 2018.

\title{
Electronics and photonics united
}

A method for integrating photonic devices with state-of-the-art nanoelectronics overcomes previous limitations. The approach shows promise for realizing high-speed, low-power optoelectronic technology. SEE LETTER P.349

\section{GORAN Z. MASHANOVICH}

$\mathrm{T}$ The integration of electronic and photonic circuits on a single silicon chip could enable unprecedented functions and performance in computing, communications and sensing at a low cost. But this goal has been hindered by the fact that most electronic circuits use bulk silicon substrates, whereas photonic circuits typically require siliconon-insulator platforms. On page 349, Atabaki et al. ${ }^{1}$ report the first fabrication of photonic devices on a bulk silicon substrate, together with millions of electronic devices known as transistors. The work paves the way for the mass production of optoelectronic systems on chips.

Photonics is prevalent in almost every aspect of day-to-day life - from smartphones and display screens to lighting and medical devices. It is often considered to be the 'electronics of the twenty-first century'. Although silicon is not an ideal photonics material (for example, lasers cannot be built from silicon), many factors have made it the main candidate for applications that require large numbers of photonic devices ${ }^{2}$. These factors include the high natural abundance of silicon, its widespread use in electronics, its optical transparency over a wide range of wavelengths and the availability of silicon-fabrication facilities that are used in micro- and nanoelectronics.

Thanks to intense research activity over the past 15 years, there have been many breakthroughs in the field of silicon photonics. Examples include hybrid silicon lasers ${ }^{3}$, various types of modulator ${ }^{4}$ (devices that convert electronic information into optical signals), high-speed light detectors ${ }^{5}$ (photodetectors) and complex optoelectronic circuits ${ }^{6}$. Several companies currently sell products based on silicon photonics chips, and many more are poised to do so in the near future.

In the electronics industry, complementarymetal-oxide-semiconductor (CMOS) technology is used to create computer processors and memory, communication chips and image sensors. This technology is based on silicon and depends on the ability to cram a large number of transistors and electronic circuits on to a single chip. Similarly, the integration of large numbers of electronic and photonic circuits on a single chip is crucial for meeting the requirements of computer processors and communication links in data centres, in terms of data-transmission rates, power consumption, scalability and complexity.

The main challenge for such integration has been the incompatibility of the material platforms used in silicon electronics and photonics. CMOS technology uses either bulk silicon substrates or thin silicon-on-insulator wafers ${ }^{6}$. The former is by far the most dominant platform because of its abundant supply chain and low cost. By contrast, silicon photonics usually requires thick silicon-on-insulator wafers that have a limited supply chain and are too expensive for many applications, such as computer memory. A long-term goal has therefore been to integrate electronic and photonic components using standard CMOSmanufacturing techniques and material platforms, without affecting the performance of such components.

Atabaki and colleagues have made a breakthrough in this regard by decoupling the formation of photonic devices from that of transistors, and by successfully incorporating these photonic devices into bulk silicon CMOS chips. The authors used standard CMOS-manufacturing methods, and introduced only a few changes to the fabrication process to create areas dedicated to photonic devices in the bulk silicon. The devices were integrated during the processing of the transistors. This involved the addition of isolated patches (islands) of the insulator material silicon dioxide to the bulk silicon and the deposition of a thin film of polycrystalline silicon on 


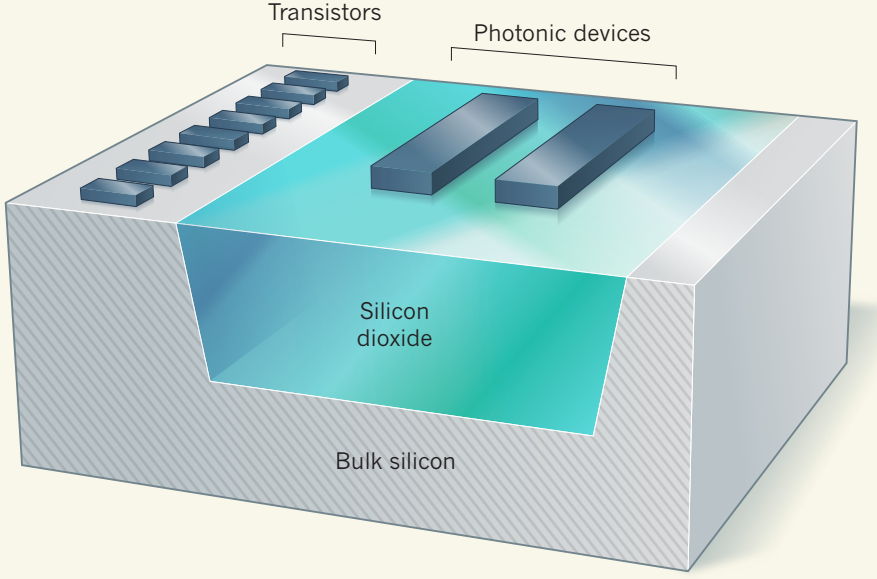

Figure 1 | Optoelectronic integration. Atabaki et al. ${ }^{1}$ report a technique for integrating electronic and photonic devices on a single silicon microchip. The authors added isolated patches (islands) of the insulator material silicon dioxide to a bulk silicon substrate - for simplicity, a single island is shown here. They then deposited a thin film of polycrystalline silicon on top. Photonic devices and electronic devices known as transistors were fabricated from this film; the former in the silicon-on-insulator region and the latter in the bulk silicon. (Adapted from Fig. 1b of ref. 1.)

top. Photonic devices were fabricated in this silicon-on-insulator region, whereas transistors were formed in standard bulk silicon regions on the CMOS chip (Fig. 1).

Although the electronic and photonic properties of crystalline silicon are superior to those of polycrystalline silicon, because the former has a more uniform structure, it is not possible to grow crystalline silicon on top of silicon dioxide. Atabaki et al. therefore opted for polycrystalline silicon, which is relatively cheap and readily available because it is used in transistor fabrication. The authors used this material to create various photonic components, including waveguides (structures that enable light propagation on chips), optical filters known as micro-ring resonators, vertical grating couplers (for coupling light between waveguides and optical fibres), high-speed modulators and photodetectors.

The performance of these components was similar to or better than previous demonstrations in polycrystalline silicon ${ }^{7-9}$. But, more importantly, the performance was unaffected by the fact that such components operated next to electronic-circuit blocks composed of millions of CMOS transistors. Consequently, the authors' silicon-photonics chips can achieve many of the goals of systems that require multiple chips, with substantial cost, scalability and performance advantages.

Atabaki and colleagues' results are impressive, but there are several aspects that could be improved. For example, optical loss in the waveguides could be reduced, and the filtering of light in the micro-ring resonators and the coupling efficiency of the vertical grating couplers could be increased. The authors suggest that optical loss might be minimized by refining the polishing process that they used to reduce the roughness of the silicon dioxide islands and the polycrystalline-silicon film. Such an improvement would lead to chips that have better photodetector sensitivity, lower voltage requirements and lower power consumption - all of which are crucial for the realization of efficient on-chip optoelectronic systems.

Further optimization of the polycrystalline-silicon film could enhance the speed of the modulators and photodetectors, which is paramount for future optical connections that can transmit data at rates of multi-terabytes per second. Atabaki et al. fabricated their silicon photonics chips using a technology based on 65-nanometre transistors, and it will be interesting to see whether their approach can be extended to smaller scales at which an even greater density of transistors can be integrated. Future work could also examine how the approach could be used for optical connections inside microprocessors.

Although there are several challenges to be overcome, the authors' work is a milestone on the path towards the mass production of onchip optoelectronic systems. We can expect an exciting period of development of such systems, and their demonstration for a host of applications. In the future, they might be as ubiquitous as today's electronic microchips.

Goran Z. Mashanovich is in the

Optoelectronics Research Centre, Faculty of

Engineering and Physical Sciences, University

of Southampton, Southampton SO17 1BJ, UK.

e-mail:g.mashanovich@soton.ac.uk

1. Atabaki, A. H. et al. Nature 556, 349-354 (2018).

2. Rickman, A. Nature Photon. 8, 579-582 (2014).

3. Zhou, Z., Yin, B. \& Michel, J. Light Sci. Appl. 4, e358 (2015).

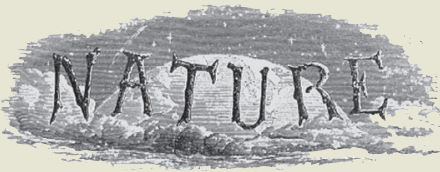

50 Years Ago

A modern approach to the study of salmon migration has been started ... The project involves the use of a computer in a three year analysis of the factors involved in the migration of salmon to fresh-water rivers and those which might affect the fish on its return to the sea. The analysis appears to be the first of its kind in Europe and possibly in the world ... Data from a number of rivers will be fed into the computer at intervals of about a month. Physical factors which may be connected with the movement of salmon are being recorded, particularly river flow, air and water temperature, amount of light ... and solar radiation ... In addition, special fish traps record all the fish swimming up or down the river. From Nature 20 April 1968

100 Years Ago

The possibility of an aerial mail has often been commented upon ... and it is very interesting to note that a company has actually been formed in Norway for the purpose of establishing a mail service between Aberdeen and Stavanger. This trip was made just before war broke out ... in about five hours' flying, and it is estimated that the mail services will reduce this to four and a half hours with modern machines. An extension of the system to Christiania and Copenhagen is contemplated, and it is hoped that letters leaving Aberdeen in the morning would be delivered in both these cities in the afternoon ... The value of such a mail service would be very great at a time when the oversea service is so seriously hampered by the German submarine campaign, and the satisfactory establishment of the contemplated Norwegian service would undoubtedly soon lead to a general use of the aeroplane for rapid international communication. From Nature 18 April 1918 
4. Thomson, D. et al. J. Opt. 18, 073003 (2016).

5. Vivien, L. et al. Opt. Express 20, 1096-1101 (2012).

6. Sun, C. et al. Nature 528, 534-538 (2015).

7. Sun, C. et al. IEEE J. Solid-State Circuits 50, 828-844 (2015).
8. Preston, K., Manipatruni, S., Gondarenko, A., Poitras, C. B. \& Lipson, M. Opt. Express 17, 5118-5124 (2009).

9. Mehta, K. K. et al. Opt. Lett. 39, 1061-1064 (2014).

\section{A stockpile of antiviral defences}

\section{The full list of weapons used by bacteria against viruses is not known. A computational approach has uncovered nine previously unidentified antiviral systems, encoded by genes near known defence genes in bacterial genomes.}

\section{SÉBASTIEN LEVESQUE \& SYLVAIN MOINEAU}

$\mathrm{F}$ lighting viruses is no easy task. Bacteria have survived attacks by viruses called phages by evolving sophisticated defence strategies that enable them to thrive even in virus-rich ecosystems. However, phages have evolved counter-tactics to thwart such mechanisms ${ }^{1}$, leading to a biological arms race. Now Doron et al. ${ }^{2}$ report the identification of previously unknown antiviral systems in bacteria.

Anti-phage systems usually target key steps in viral replication. For example, some systems prevent phage binding to bacterial cells, whereas others block entry of the viral genome into the cell ${ }^{3}$. Certain bacterial proteins can halt intracellular phage replication ${ }^{3,4}$. Although this often leads to the death of infected cells, it can protect neighbouring cells from infection. Perhaps the best known anti-phage systems are restriction enzymes and CRISPR-Cas. These two systems ${ }^{5-7}$, can cleave non-host DNA in a sequence-specific manner, and have also been widely adapted as molecular tools in the biological sciences.

As knowledge of the diversity of Earth's viruses has grown ${ }^{8}$, along with the potential of using such information to develop further biotechnology tools, investigation into anti-phage systems has surged. Many lines of evidence have indicated that the list

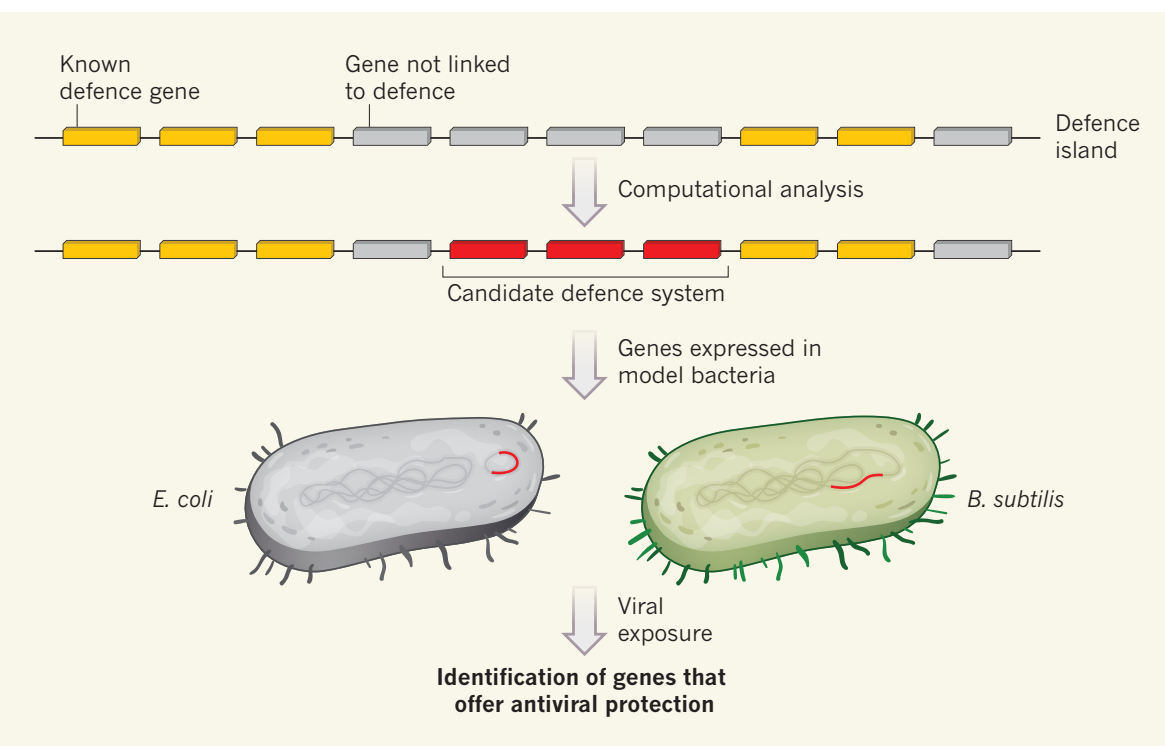

Figure 1 | Identifying antiviral systems in bacteria. Bacterial defence genes (yellow) are found in regions of the genome known as defence islands. Doron et al. ${ }^{2}$ sought to identify more such genes by analysing genes within these islands that had not previously been linked to defence functions (grey). They used computational analysis involving a range of criteria, including whether the genes were located in defence islands in many different types of bacterium. The authors also identified neighbouring genes that might function together as a defence system. These proposed defence-system genes were then expressed in the model bacteria Escherichia coli and Bacillus subtilis. The bacteria were exposed to various viruses to test whether the genes offered protection against infection. The authors confirmed that nine of the defence systems they tested had antiviral functions. 